\title{
葫芦腿的分解氧化研究
}

\author{
路红燕史大昕张奇杨德利李加荣* \\ (北京理工大学化工与环境学院 北京 100081)
}

\begin{abstract}
摘要 葫芦脲是高度对称的刚性分子, 在试图改进 Kim 等氧化制取羟基葫芦脲的过程中发现, 葫芦 [6]艮(CB[6]) 在多 种过硫酸盐(过硫酸钾、过硫酸钠、过硫酸胺) 的氧化下, 除了可以获得羟基葫芦脲外, 还可以得到进一步分解产物草 酸. 研究表明, $\mathrm{CB}[n](n=5,7,8)$ 及其差基葫芦䐂也存在类似的现象. 这是国内外首次发现葫芦脲在进行腰位 $\mathrm{C}-\mathrm{H}$ 键 着基化的同时, 还可以进一步氧化形成草酸.
\end{abstract}

关键词 葫芦脲; 羟基葫芦脲; 氧化; 草酸

\section{Oxidation of Cucurbituril and Its Analogues}

\author{
Lu, Hongyan Shi, Daxin Zhang, Qi Yang, Deli Li, Jiarong* \\ (School of Chemical Engineering and Environment, Beijing Institute of Technology, Beijing 100081)
}

\begin{abstract}
Cucurbituril is a high symmetry and rigid structure. When cucurbit[6]uril (CB[6]) was oxidized with persulfate (e.g. potassium persulfate, sodium persulfate and ammonium persulfate), the main product was perhydroxy-cucurbituril and the minor one was oxalic acid. Oxalic acid was also obtained by the oxidization of both $\mathrm{CB}[n](n=5,7,8)$ and perhydrox-cucurbituril with various persulfates. As far as we know, this is the first time to discover that both cucurbit[n]uril and perhydrox-cucurbit $[n]$ uril can be destroyed to give a small molecule-oxalic acid.
\end{abstract}

Keywords cucurbituril; perhydroxy-cucurbituril; oxidation; oxalic acid

葫芦腿是一个具有笼状对称结构的大环含氮化合 物, 最常见的是含六个甘脲单元的葫芦脲(简写为 $\mathrm{CB}[6]) . \mathrm{CB}[6]$ 早在 1905 年就由德国化学家 Behrend等合 成出来 ${ }^{[1]}$, 但直到 20 世纪 80 年代前后德国另一位学者 Freeman 和美国学者 Mock 等重新研究了 Behrend 等的 工作, 并用硫酸钙处理获得缩合产物与钲离子络合的水 合体，再通过结构测定确定其缩合产物是由六个甘嫝经 由亚甲基桥联的大环聚合物 $\mathrm{CB}[6]^{[2]}$. 迄今, 一系列 $\mathrm{CB}[6]$ 的类似物, 如 $\mathrm{CB}[5], \mathrm{CB}[7], \mathrm{CB}[8], i \mathrm{CB}[6], i \mathrm{CB}[7]$,

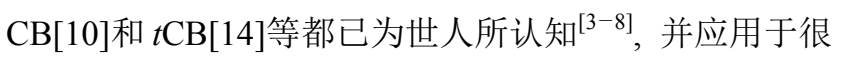
多方面 ${ }^{[0 \sim 11]}$.

葫芦嫝是甘䐂经由亚甲基桥联而成的高度对称的 中空刚性分子, 具有两个环绕的酰胺羰基氧原子组成的 亲水性端口和一个疏水性腔体的笼体结构.葫芦腿包含 三个结构单元: (1)腿基; (2)腰位偕二次甲基 $\mathrm{C}-\mathrm{H}\left(\mathrm{R}^{1}\right)$ 和(3)桥联亚甲基 $\mathrm{CH}_{2}$ (或 $\mathrm{CHR}_{2}$ ) (图 1). 理论上这三部分 都可以发生反应(或转化), 即腰位偕二次甲基 $\mathrm{C}-\mathrm{H}$ 键
和桥联亚甲基 $\mathrm{C}-\mathrm{H}$ 键的功能化; 腿羰基的还原或水解 (Scheme 1), 特别是酰胺一般易水解 ${ }^{[12]}$, 但葫芦腿的弧 形偕环结构使得其非常稳定, 能够耐受 $370{ }^{\circ} \mathrm{C}$ 的高温 而不分解，即使是 $100^{\circ} \mathrm{C}$ 的浓盐酸, $\mathrm{CB}[5], \mathrm{CB}[6], \mathrm{CB}[7]$ 也不分解 ${ }^{[4]}$.

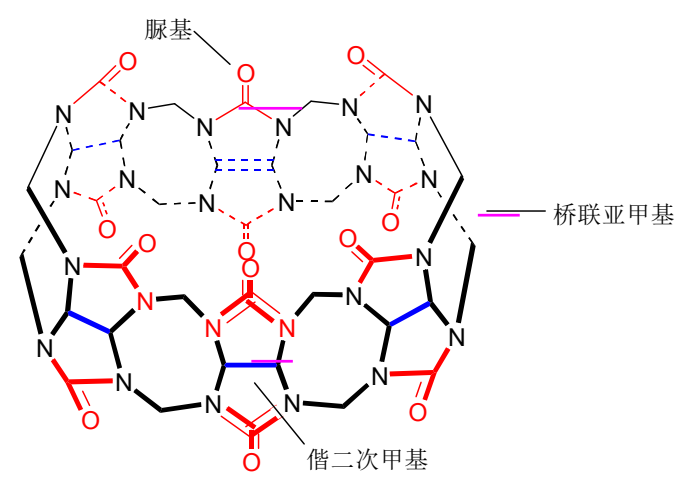

图 $1 \mathrm{CB}[6]$ 可能的反应部位

Figure 1 Possible reaction position of $\mathrm{CB}[6]$

\footnotetext{
* E-mail: jrli@bit.edu.cn

Received September 3, 2014; revised October 27, 2014; published online November 5, 2014.

Project supported by the Basic Research Fund of Beijing Institute of Technology (No. 20131042006).

北京理工大学基础研究基金(No. 20131042006)资助项目..
} 


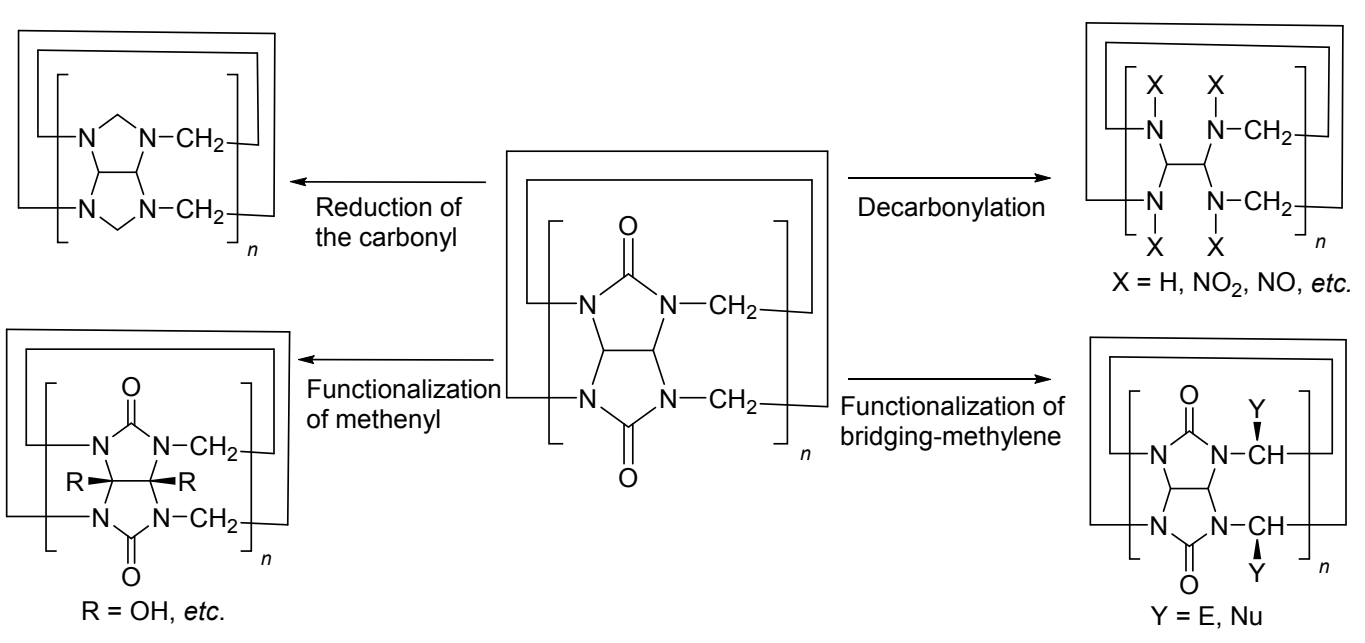

图式 $1 \mathrm{CB}[6]$ 反应类型

Scheme 1 Possible reaction type of $\mathrm{CB}[6]$

(a) Reduction of the carbonyl; (b) decarbonylation; (c) functionalization of methenyl; (d) functionalization of bridging-methylene

迄今为止, 人们已经合成了一些葫芦艮衍生物, 其 中腰位偕二次甲基功能化衍生物有全取代的十甲基葫 芦[5]腿 $\left(\mathrm{Me}_{10} \mathrm{CB}[5]\right)$ 、环己基葫芦[5]脲 $\left(\mathrm{Cy}_{5} \mathrm{CB}[5]\right)$ 、环己 基葫芦 [6]脲 $\left(\mathrm{Cy}_{6} \mathrm{CB}[6]\right.$ )和部分取代的二苯基葫芦 [6]嫝 $\mathrm{Ph}_{2} \mathrm{CB}[1,5]$ 、甲基苯基葫芦 [7]艮( $\mathrm{PhMeCB}[1,6]$ )、六甲基 葫芦 [6]嫝 $\left(\mathrm{Me}_{6} \mathrm{CB}[3,3]\right)^{\text {等 }}{ }^{[13 \sim 15]}$; 桥联亚甲基功能化单芳 基 $\mathrm{CB}[6]$ 、单甲基或丙基取代 $\mathrm{CB}[6]$ ，以及上下两个亚甲 基同时功能化产物等 ${ }^{[16 ~ 18]}$.

这些化合物的合成无一例外均采用甘腿衍生物或 甲酫衍生物作为起始物, 而不是经由 $\mathrm{C}-\mathrm{H}$ 键的功能化 转化而成. 由葫芦豚为底物进行直接衍生化成功的例子 只有 2003 年韩国学者 Kim 以及 2012 年英国学者 Scherman 等实施的羟基化 ${ }^{[19,20]}$. 我们在相关的羟基化研 究中发现 $\mathrm{CB}[6]$ 及其同系物等可以方便地氧化成草酸, 这是国内外首次报道葫芦脲可氧化分解获得小分子产 物.

\section{1 结果与讨论}

韩国学者 Kim 等报道的 12 羟基葫芦嫝的制备是采 用过量的过硫酸钾 $\left(\mathrm{K}_{2} \mathrm{~S}_{2} \mathrm{O}_{8}, \mathrm{KPS}\right)$ 氧化葫芦脲分子. 英国 学者 Scherman 等人在用咪唑离子液体增加 $\mathrm{CB}[6]$ 在水 中的溶解性及将氧化剂改为室温溶解性好的过硫酸铵 $\left(\left(\mathrm{NH}_{4}\right)_{2} \mathrm{~S}_{2} \mathrm{O}_{8}, \mathrm{APS}\right)$ 后, 采用等量的物料反应成功地制得 了单羟基化产物(Scheme 2).

这个反应的机理目前大都认为是自由基氧化, 我们 尝试使用其它一系列常用的氧化剂如双氧水、芬顿试 剂、过氧乙酸、APS、四甲基哌啶氧化物自由基等改进 其转化, 效果均不明显. 在重复 Kim 的氧化条件氧化 $\mathrm{CB}[6]$ 时，所制得的羟基葫芦腿的 MALDI-TOF 质谱中 未发现文献报道的 $(\mathrm{HO}){ }_{12} \mathrm{CB}[6]$ 的 $\left[\mathrm{M}+\mathrm{K}^{+}\right]$峰 $(m / z=$
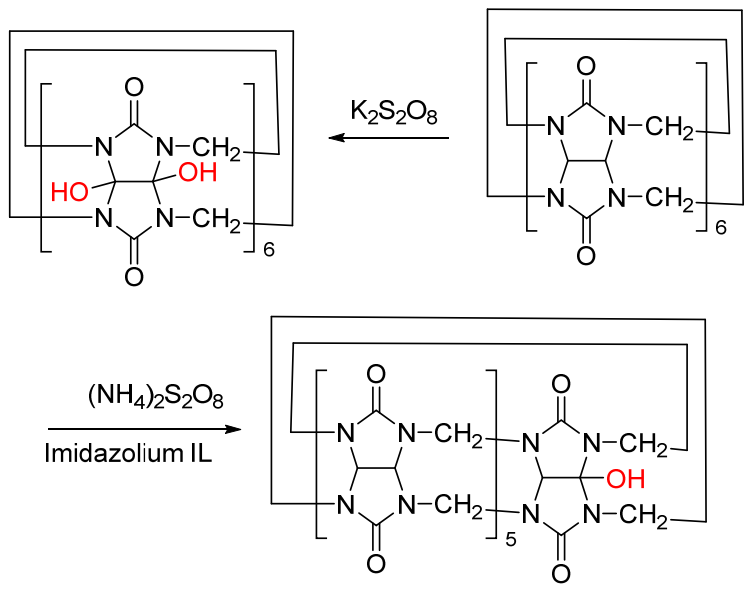

图式 2 文献已报的 $\mathrm{CB}[6]$ 全羟基化和单羟基化

Scheme 2 Full-hydroxylation and mono-hydroxylation of $\mathrm{CB}[6]$ reported in literature

$1227.1)$ 和 $\left[\mathrm{M}+2 \mathrm{~K}^{+}\right]$峰 $(m / z=1266.1)$, 仅看到 $[\mathrm{CB}[6]+$ $\left.\mathrm{K}^{+}\right]$峰 $(m / z=1035.3) 、\left[(\mathrm{HO})_{1} \mathrm{CB}[6]+\mathrm{K}^{+}\right]$峰 $(m / z=$ 1051.3)、 $\left[(\mathrm{HO})_{2} \mathrm{CB}[6]+\mathrm{K}^{+}\right]$峰 $(m / z=1067.3) 、\left[(\mathrm{HO})_{3}{ }^{-}\right.$ $\left.\mathrm{CB}[6]+\mathrm{K}^{+}\right]$峰 $(m / z=1083.3)$ 和 $\left[(\mathrm{HO})_{4} \mathrm{CB}[6]+\mathrm{K}^{+}\right]$峰 $(m / z=1099.3)$ (图 2). 这表明在文献的氧化条件下未能 得到全羟基取代的葫芦腿( $\mathrm{HO})_{12} \mathrm{CB}[6]$.

鉴于以上实验结果，我们将氧化条件调整为: 氧化 剂用量加大至 $\mathrm{C}-\mathrm{H}$ 键反应位点的 3.6 倍(文献用量为 1.2 倍)、温度由 $85{ }^{\circ} \mathrm{C}$ 升高至回流、反应时间从 $6 \mathrm{~h}$ 延长 至 $24 \mathrm{~h}$. 反应结束后体系冷却至室温, 析出的固体用甲 醇进行索氏提取, 所得到的甲醇溶液浓缩, 浓缩液静置 析出晶体. 该晶体经 $\mathrm{X}$ 射线单晶衍射检测为含有结晶水 的草酸(图 3).

毫无疑问，草酸应来至于 $\mathrm{CB}[6]$ 的腰间偕二次甲基 的氧化所得(Eq. 1). 


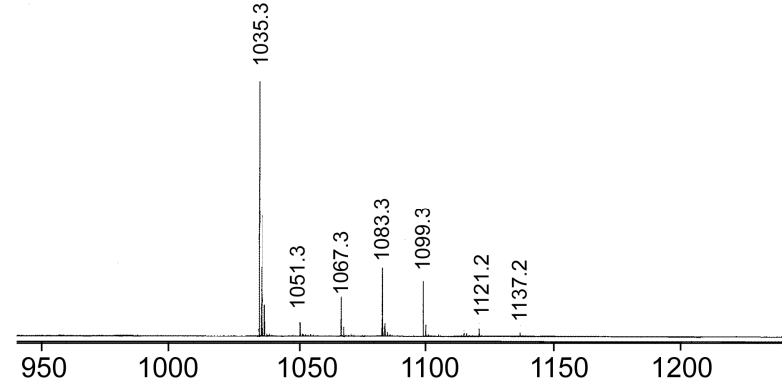

图 2 葫芦艮 $[6]$ 过硫酸钾氧化产物

Figure 2 The oxidation product of $\mathrm{CB}[6]$ by KPS
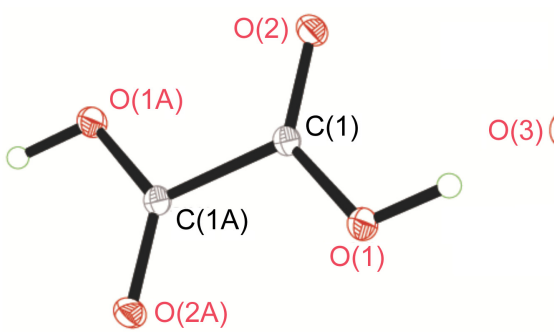

图 3 含有结晶水的草酸单晶图

Figure 3 X-ray crystal structure of oxalic acid

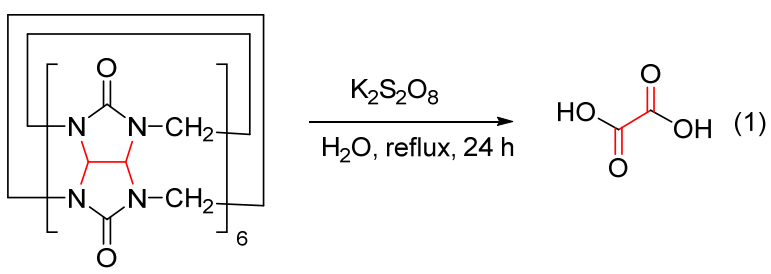

值得指出的是, 在 Kim 氧化条件下经改进后得到羟 基葫芦嫝的残液中, 也可以得到少量草酸; 且将氧化剂 换为 $\mathrm{Na}_{2} \mathrm{~S}_{2} \mathrm{O}_{8}$ (NPS)或 APS, 葫芦脲 $\mathrm{CB}[6]$ 也可以被氧化 成着基葫芦脲及草酸(Eq. 2).

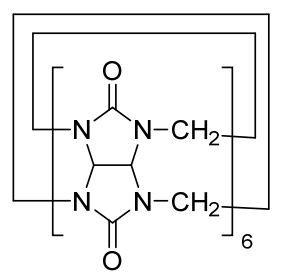

$$
\frac{[\mathrm{O}]}{75^{\circ} \mathrm{C}, 50^{\circ} \mathrm{C}, 16 \mathrm{~h}}
$$

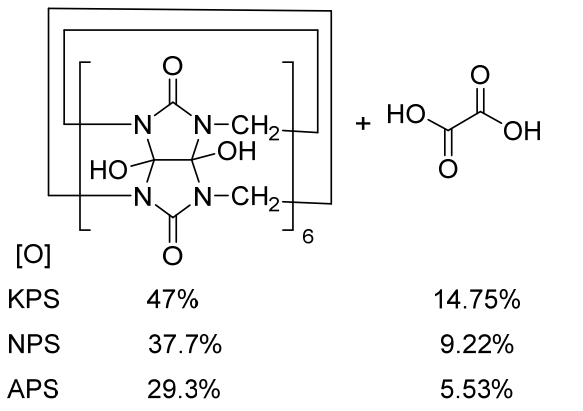

同样地，当我们用该条件氧化 $\mathrm{CB}[6]$ 的同系物 $\mathrm{CB}[n](n=5,7,8)$ 及羟基葫芦嫝时也能明确得到草酸
(Eq. 3).

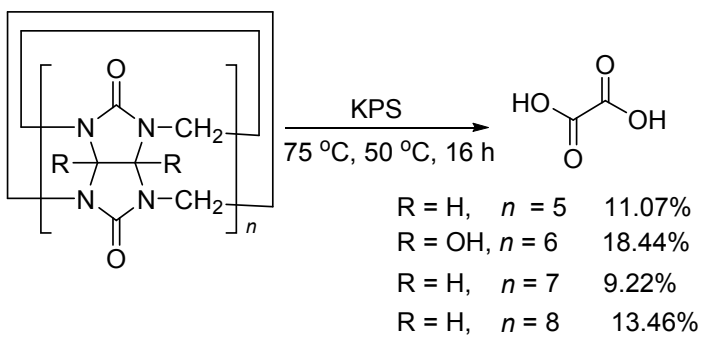

有意思的是，当我们用葫芦腿的前体甘腿作底物， 在上述氧化条件下检验其是否也能氧化成草酸时发现, 产物不是草酸而是氧化成咪唑三酮(Scheme 3). 该产物 的结构得到 IR, HNMR, MS 的表征, 也为单晶测定所证 实(图 4).

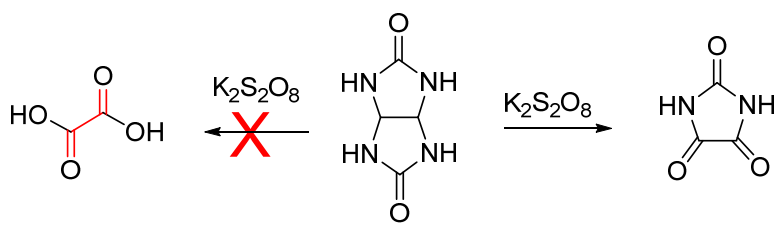

图式 3 甘腿的氧化

Scheme 3 The oxidation of glycoluril

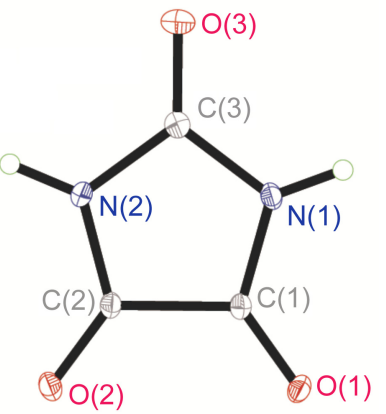

图 4 咪唑三酮的单晶图

Figure 4 X-ray crystal structure of imidazolidine-2,4,5-trione

\section{2 结论}

在剖析葫芦脲骨架及其可能反应的基础上，首次开 展了葫芦腿及其衍生物的分解氧化研究, 发现 $\mathrm{CB}[n]$ $(n=5,6,7,8)$ 及羟基葫芦嫝均可在过硫酸盐氧化剂作用 下部分氧化成草酸.

\section{3 实验部分}

\section{1 仪器与试剂}

过硫酸钾 $\mathrm{K}_{2} \mathrm{~S}_{2} \mathrm{O}_{8}$ (KPS)、过硫酸铵 $\left(\mathrm{NH}_{4}\right)_{2} \mathrm{~S}_{2} \mathrm{O}_{8}$ (APS) 和过硫酸钠 $\mathrm{Na}_{2} \mathrm{~S}_{2} \mathrm{O}_{8}$ (NPS)购自北京化学试剂公司, 其 它的试剂购自不同商家, 均是分析纯, 水为蒸馏水. 熔 点测定使用 XT4 显微熔点测定仪(未校正); 红外光谱用 
Thermo Fisher Scientific Nicolet iS10 型傅立叶变换型红 外仪 $(\mathrm{KBr}$ 压片)测定; 核磁共振氢谱采用 Varian mercury-plus 400 型核磁共振仪记录, DMSO- $d_{6}$ 为溶剂, TMS 为内标; ESI 质谱用 Varian 500-MS 型液-质联用仪测定; 元素分析由 ElementarVarioEL 测定; 晶体结构用 Bruker Smart 1000 CCD X 射线四圆衍射仪测定.

\section{2 实验方法}

\subsection{1 葫芦腿的合成}

$\mathrm{CB}[n](n=5,6,7,8)$ 的合成参照文献[21, 22]制得.

$\mathrm{CB}[5]$ : $30 \mathrm{~g}$, 产率 23.1\%; m.p. $>300{ }^{\circ} \mathrm{C}$; ${ }^{1} \mathrm{H} \mathrm{NMR}$ $\left(\mathrm{D}_{2} \mathrm{SO}_{4} / \mathrm{D}_{2} \mathrm{O}, 400 \mathrm{MHz}\right) \delta: 3.29(\mathrm{~d}, J=16.0 \mathrm{~Hz}, 12 \mathrm{H}), 2.61$ (s, 12H), 1.35 (d, $J=16.0 \mathrm{~Hz}, 12 \mathrm{H})$; IR (KBr) v: 3445, 2999, 2937, 1739, 1638, 1477, 1417, 1377, 1328, 1293, $1233,1190,964,809,795,765,673,627 \mathrm{~cm}^{-1}$.

$\mathrm{CB}[6]$ : $35 \mathrm{~g}$, 产率 26.9\%; m.p. $>300{ }^{\circ} \mathrm{C} ;{ }^{1} \mathrm{H}$ NMR $\left(\mathrm{D}_{2} \mathrm{SO}_{4} / \mathrm{D}_{2} \mathrm{O}, 400 \mathrm{MHz}\right) \delta: 2.73(\mathrm{~d}, J=16.0 \mathrm{~Hz}, 10 \mathrm{H}), 3.17$ (s, 10H), $1.90(\mathrm{~d}, J=16.0 \mathrm{~Hz}, 10 \mathrm{H})$; IR (KBr) v: 3441, 2998, 2932, 1735, 1475, 1416, 1376, 1326, 1295, 1234, $1190,1147,964,801,758,673,628 \mathrm{~cm}^{-1}$.

$\mathrm{CB}[7]$ : $23 \mathrm{~g}$, 产率 17.7\%; m.p. $>300{ }^{\circ} \mathrm{C} ;{ }^{1} \mathrm{H}$ NMR $\left(\mathrm{D}_{2} \mathrm{SO}_{4} / \mathrm{D}_{2} \mathrm{O}, 400 \mathrm{MHz}\right) \delta: 2.76(\mathrm{~d}, J=16.0 \mathrm{~Hz}, 14 \mathrm{H}), 2.61$ (s, 14H), 1.32 (d, $J=16.0 \mathrm{~Hz}, 14 \mathrm{H})$; IR (KBr) v: 3445, 2999, 2934, 1732, 1476, 1420, 1376, 1325, 1293, 1234, $1191,967,825,806,759,674,628 \mathrm{~cm}^{-1}$.

$\mathrm{CB}[8]: 10.15 \mathrm{~g}$, 产率 7.8\%; m.p. $>300{ }^{\circ} \mathrm{C}$; IR (KBr) $v: 3445,3002,2925,1725,1474,1425,1375,1319,1293$, $1231,1191,1157,993,970,830,808,759,675,631 \mathrm{~cm}^{-1}$.

\section{$3.2 .2 \mathrm{CB}[6]$ 的氧化}

将 $\mathrm{CB}[6](1.0 \mathrm{~g}, 1.0 \mathrm{mmol}) 、 \mathrm{~K}_{2} \mathrm{~S}_{2} \mathrm{O}_{8}(3.9 \mathrm{~g}, 14.4$ $\mathrm{mmol}$ )加入含有 $50 \mathrm{~mL}$ 蒸馏水的圆底烧瓶中, 用保护气 $\mathrm{N}_{2}$ 鼓泡, 温度升至 $75{ }^{\circ} \mathrm{C}$. 纯清后降温至 $50{ }^{\circ} \mathrm{C}$ 搅拌 16 $\mathrm{h}$, 冷却, 室温滤除杂质. 滤液浓缩至 $10 \mathrm{~mL}$, 将浓缩液 滴加至丙酮 $(150 \mathrm{~mL})$ 中析出白色固体, 过滤得粗品, 粗 品用 $6 \mathrm{~mL}$ DMSO 溶解. 抽滤, 滤液缓慢滴至丙酮中析 出纯品 $(\mathrm{HO})_{12} \mathrm{CB}[6](580 \mathrm{mg})$, 产率 47\%. m.p. $>300{ }^{\circ} \mathrm{C}$; ${ }^{1} \mathrm{H}$ NMR (DMSO- $\left.d_{6}, 400 \mathrm{MHz}\right) \delta: 4.16 \sim 4.49(\mathrm{~m}, 12 \mathrm{H})$, $5.17 \sim 5.54(\mathrm{~m}, 12 \mathrm{H}), 7.95(\mathrm{~s}, 12 \mathrm{H})$; IR $(\mathrm{KBr}) v: 3456$, 3206, 3013, 2952, 1740, 1473, 1420, 1376, 1323, 1262, 1201, 1049, 965, 799, 762, 670, $584 \mathrm{~cm}^{-1}$; MS (ESI) $\mathrm{m} / \mathrm{z}$ (\%): $1193\left(\mathrm{M}-\mathrm{H}_{2} \mathrm{O}+\mathrm{K}^{+}, 95\right), 616\left(\mathrm{M}-\mathrm{H}_{2} \mathrm{O}+2 \mathrm{~K}^{+}\right.$, 100). Anal. calcd for $\mathrm{C}_{36} \mathrm{H}_{36} \mathrm{~N}_{24} \mathrm{O}_{24} \bullet 8 \mathrm{H}_{2} \mathrm{O}: \mathrm{C} 32.44$, H 3.93, N 25.22; found C 32.28, H 3.94, N 25.34.

滤除粗品后的滤液在 $50{ }^{\circ} \mathrm{C}$ 浓缩, 浓缩液放置一天 后即可析出固体. 该固体用甲醇进行索氏提取, 浓缩得 草酸(0.08 g), 产率 $14.75 \%$; m.p. $180 \sim 182{ }^{\circ} \mathrm{C}$; IR (KBr) $v: 3476,1647,1234,1124,724,619 \mathrm{~cm}^{-1}$.

草酸溶解在甲醇及水的混合液中，适量丙酮慢慢扩 散进去，从而得到草酸的单晶，选取尺寸约为 0.58 $\mathrm{mm} \times 0.52 \times 0.47 \mathrm{~mm}$ 适当的晶体, 用 Bruker Smart Apex 单晶衍射仪, 采用经石墨单色器单色化的 Mo K $\alpha$ 射线( $\lambda$ $=0.071073 \mathrm{~nm})$, 于 $153(2) \mathrm{K}$ 下，以 $\varphi-\omega$ 扫描方式收集 单晶衍射数据. 强度数据进行了经验吸收校正、 $\mathrm{Lp}$ 校正, 晶体结构由直接法解得，对全部非氢原子坐标及其各向 异性热参数进行了全矩阵最小二乘法修正，所有计算用 SHELX-97 程序完成. 图 3 为其单晶图, 分子式为 $\left(\mathrm{C}_{2} \mathrm{H}_{2} \mathrm{O}_{4}\right) \cdot 2 \mathrm{H}_{2} \mathrm{O}$, 相对分子质量为 126.07 , 单斜晶系, 空 间群 $P 2(1) / n$, 晶胞参数为: $a=0.6101(4) \mathrm{nm}, b=0.3529$ (2) $\mathrm{nm}, c=1.1855(7) \mathrm{nm}, \alpha=90^{\circ}, \beta=103.745(7)^{\circ}, \gamma=$ $90^{\circ}$, 体积 $V=0.2479(2) \mathrm{nm}^{3}, D_{\mathrm{c}}=1.689 \mathrm{mg} / \mathrm{m}^{3}, F(000)=$ 132. 晶胞中分子数为 2 , 吸收系数 $\mu=1.79 \mathrm{~cm}^{-1}$.

\section{2 .3 甘㽷的氧化}

将甘脲 $(1.0 \mathrm{~g}) 、 \mathrm{~K}_{2} \mathrm{~S}_{2} \mathrm{O}_{8}(3.9 \mathrm{~g}, 14.4 \mathrm{mmol})$, 加入含有 $50 \mathrm{~mL}$ 蒸馏水的圆底烧瓶中，用保护气 $\mathrm{N}_{2}$ 鼓泡，温度逐 渐升至 $75{ }^{\circ} \mathrm{C}$, 反应 $16 \mathrm{~h}$. 反应结束后将反应液冷至室 温, 乙酸乙酯萃取, 萃取液挥发干后即可得到咪唑三酮. 产率 95\%. m.p. $240{ }^{\circ} \mathrm{C} ;{ }^{1} \mathrm{H}$ NMR (400 MHz, DMSO- $d_{6}$ ) $\delta_{\mathrm{H}}: 11.75(\mathrm{br}, 2 \mathrm{H})$; IR (KBr) $v: 3052,2708,2488,1745$, 1377, 1341, 1230, $993 \mathrm{~cm}^{-1}$; MS (ESI) $m / z(\%): 114\left(\mathrm{M}^{+}\right.$, 100).

咪唑三酮溶解在乙酸乙酯中, 慢慢挥发从而得到三 酮的的单晶，选取尺寸约为 $0.42 \mathrm{~mm} \times 0.28 \mathrm{~mm} \times 0.10$ $\mathrm{mm}$ 的晶体，用 Bruker Smart Apex 单晶衍射仪，采用经 石墨单色器单色化的 Mo K $\alpha$ 射线 $(\lambda=0.071073 \mathrm{~nm})$, 于 153(2) K 下，以 $\varphi-\omega$ 扫描方式收集单晶衍射数据，强度 数据进行了经验吸收校正、 $\mathrm{Lp}$ 校正, 晶体结构由直接法 解得, 对全部非氢原子坐标及其各向异性热参数进行了 全矩阵最小二乘法修正，所有计算用 SHELX-97 程序完 成. 图 4 为其单晶图, 分子式为 $\mathrm{C}_{3} \mathrm{H}_{2} \mathrm{~N}_{2} \mathrm{O}_{3}$, 相对分子质 量为 114.07 , 单斜晶系, 空间群 $P 2(1) / n$, 晶胞参数为: $a=0.49822(19) \mathrm{nm}, b=0.8189$ (3) nm, $c=1.0698(4) \mathrm{nm}$, $\alpha=90^{\circ}, \beta=92.335(5)^{\circ}, \gamma=90^{\circ}$, 体积 $V=0.4361(3) \mathrm{nm}^{3}$, $D_{\mathrm{c}}=1.737 \mathrm{mg} / \mathrm{m}^{3}, F(000)=232$. 晶胞中分子数为 4 , 吸 收系数 $\mu=1.58 \mathrm{~cm}^{-1}$.

辅助材料(Supporting Information) 化合物的核磁、质 谱及单晶数据. 这些材料可以免费从本刊网站 (http://sioc-journal.cn/)上下载.

\section{References}

[1] Behrend, R.; Meyer, E.; Rusche, F. Liebigs Ann. Chem. 1905, 339, 1. 
[2] Freeman, W. A.; Mock, W. L.; Shih, N. Y. J. Am. Chem. Soc. 1981, 103, 7367.

[3] Kim, J.; Jung, I. S.; Kim, S. Y.; Lee, E.; Kang, J. K.; Sakamoto, S.; Yamaguchi, K.; Kim, K. J. Am. Chem. Soc. 2000, 122, 540.

[4] Day, A. I.; Arnold, A. P.; Blanch, R. J.; Snushall, B. J. Org. Chem. 2001, 66, 8094.

[5] Day, A. I.; Blanch, R. J.; Arnold, A. P.; Lorenzo, S.; Lewis, G. R.; Dance, I. Angew. Chem. Int. Ed. 2002, 41, 275.

[6] Liu, S.; Zavalij, P. Y.; Isaacs, L. J. Am. Chem. Soc. 2005, 127, 16798.

[7] Cheng, X. J.; Liang, L. L.; Chen, K.; Ji, N. N.; Xiao, X.; Zhang, J. X.; Zhang, Y. Q.; Xue, S. F.; Zhu, Q. J.; Ni, X. L.; Tao, Z. Angew. Chem. Int. Ed. 2013, 52, 7252

[8] Isaacs, L.; Park, S. K.; Liu, S.; Ko, Y. H.; Selvapalam, N.; Kim, Y.; Kim, H.; Zavalij, P. Y.; Kim, G. H.; Lee, H. S.; Kim, K. J. Am. Chem. Soc. 2005, 127, 18000.

[9] Han, C. Y.; Zhang, Z. B.; Chi, X. D.; Zhang, M. M.; Yu, G. C.; Huang, F. H. Acta Chim. Sinica 2012, 70, 1775 (in Chinese). (韩成友, 张子涁, 池小东, 张明明, 喻国灿, 黄飞鹤, 化学学报, 2012, 70, 1775.)

[10] Zheng, R.; Liang, D. X.; Yu, X. T.; Chen, R. E.; Jiang, H. J.; Zhou, Q. Z. Chin. J. Org. Chem. 2013, 33, 504 (in Chinese).

(郑睿, 梁丹霞, 于雪涛, 陈仁尔, 蒋华江, 周其忠, 有机化学, 2013, 33, 504.)

[11] Ma, M. F.; Xing, P. Y.; Li, S. Y.; Chu, X. X.; Wang, B.; Hao, A. Y. Prog. Chem. 2014, (8), 1317 (in Chinese). (马明放, 邢鹏遥, 李尚洋, 初晓晓, 王波, 郝爱友, 化学进展, 2014, (8), 1317.)

[12] (a) Boissonnas, R. A.; Blodinger, J.; Welcher, A. D. J. Am. Chem. Soc. 1952, 74, 5309.

(b) Anna, B.; Gerald, P.; Dendroamide, A. Heterocycle 2002, 58,
521.

[13] Han, B. H.; Liu, Y. Chin. J. Org. Chem. 2003, 23, 139 (in Chinese). (韩宝航, 刘育, 有机化学, 2003, 23, 139.)

[14] Lee, W.; Samal, S.; Selvapalam, N.; Kim, H. J.; Kim, K. Acc. Chem. Res. 2003, 36, 621.

[15] (a) Flinn, A.; Hough, G. C.; Stoddart, J. F.; Williams, D. J. Angew. Chem. Int. Ed. 1992, 31, 1475.

(b) Zhao, J.; Kim, H. J.; Oh, J.; Kim, S. Y.; Lee, J. W.; Sakamoto, S.; Yamaguchi, K.; Kim, K. Angew. Chem., Int. Ed. 2001, 40, 4233.

(c) Isobe, H.; Sato, S.; Nakamura, E. Org. Lett. 2002, 4, 1287.

(d) Miyahara, Y.; Abe, K.; Inazu, T. Angew. Chem., Int. Ed. 2002, 41, 3020 .

(e) Day, A. I.; Arnold, A. P.; Blanch, R. J. Molecules 2003, 8, 74.

(f) Vinciguerra, B.; Cao, L.; Cannon, J. R.; Zavalij, P. Y.; Fenselau, C.; Isaacs, L. J. Am. Chem. Soc. 2012, 134, 13133.

[16] Lucas, D.; Minami, T.; Iannuzzi, G.; Cao, L.; Wittenberg, J. B.; Jr, P. A.; Isaacs, L. J. Am. Chem. Soc. 2011, 133, 17966.

[17] Cao, L.; Isaacs, L. Org. Lett. 2012, 14, 3072.

[18] Gilberg, L.; Khan, M. S. A.; Enderesova, M.; Sindelar, V. Org. Lett. 2014, 16, 2446.

[19] Jon, S. Y.; Selvapalam, N.; Oh, D. H.; Kang, J. K.; Kim, S. Y.; Kim, K. J. Am. Chem. Soc. 2003, 125, 10186.

[20] Zhao, N.; Lloyd, G. O.; Scherman, O. A. Chem. Commun. 2012, 48, 3070.

[21] Meng, F. M.S. Thesis, Guizhou University, Guizhou, 2006 (in Chinese). (孟飞, 硕士论文, 贵州大学, 贵州, 2006.)

[22] Bi, Q.; Hu, Y. P.; Yang, Q.; Ma, C. L.; Li, D. L. Chin. J. Org. Chem. 2007, 27, 880 (in Chinese).

(毕强, 胡英鹏, 杨琴, 马彩莲, 李东亮, 有机化学, 2007, 27, 880.)

(Lu, Y.) 